\title{
Dislocation climb in two-dimensional discrete dislocation dynamics
}

\author{
Kamyar M. Davoudi, ${ }^{1}$ Lucia Nicola, ${ }^{2}$ and Joost J. Vlassak ${ }^{1, a)}$ \\ ${ }^{1}$ School of Engineering and Applied Science, Harvard University, Cambridge, Massachusetts 02138, USA \\ ${ }^{2}$ Department of Materials Science and Engineering, Delft University of Technology, Mekelweg 2, \\ 2628 CD Delft, The Netherlands
}

(Received 20 February 2012; accepted 16 April 2012; published online 23 May 2012)

\begin{abstract}
In this paper, dislocation climb is incorporated in a two-dimensional discrete dislocation dynamics model. Calculations are carried out for polycrystalline thin films, passivated on one or both surfaces. Climb allows dislocations to escape from dislocation pile-ups and reduces the strainhardening rate, especially for fully passivated films. Within the framework of this model, climb modifies the dislocation structures that develop during plastic deformation and results in the formation of pile-ups on slip planes that do not contain any dislocation sources. (C) 2012 American Institute of Physics. [http://dx.doi.org/10.1063/1.4718432]
\end{abstract}

\section{INTRODUCTION}

The development of micro-electronic and micro-electromechanical systems (MEMS) has stimulated a strong interest in the mechanical properties of materials at the micro- and nano-scales. Many experiments ${ }^{1-9}$ have revealed that the mechanical behavior of a material at small length scales can be quite different from that of the same material in bulk form. Plasticity at small scales occurs by the same basic mechanisms observed in bulk materials: plastic flow arises mainly from the collective motion of dislocations in crystalline solids. The limited availability of dislocation sources and the proximity of interfaces and free surfaces, however, result in rich plastic behavior as the length scale is reduced. In this paper, we investigate some of these effects in polycrystalline thin films passivated on one or both surfaces using a twodimensional discrete dislocation dynamics (DDD) model. The film passivated on one surface is representative of a film on a substrate where dislocations can freely escape from the surface of the film, while the film passivated on both surfaces can be regarded as a film on a substrate where a thin passivating layer prevents escape of dislocations. The discrete dislocation formulation follows that developed by Van der Giessen and Needleman, ${ }^{10}$ in which a plane-strain model of a polycrystalline film is used. Plastic flow arises from the motion of discrete edge dislocations, represented as line singularities in an elastic solid, with the long-range interactions between dislocations directly accounted for. Drag during dislocation motion, dislocation nucleation, and dislocation annihilation is incorporated through a set of constitutive rules, while the boundary conditions are enforced by solving for the image field. While the two-dimensional formulation clearly cannot capture all of the physics, it allows for the modeling of realistic numbers of dislocations and microstructures, and it has been quite successful at simulating the behavior of thin films over a range of microstructures. ${ }^{11-19}$

Most previous DDD studies ${ }^{11-25}$ have been limited to low temperatures. Since diffusion of point defects is

\footnotetext{
a) Author to whom correspondence should be addressed. Electronic mail: vlassak@seas.harvard.edu.
}

negligible at low temperatures, the movement of dislocations is limited to glide. At elevated temperatures, however, diffusion cannot be neglected and dislocation climb is observed (see for instance the references in Section 8.4 of Ref. 26). In climb, edge dislocations leave their slip planes through emission or absorption of point defects. During plastic deformation, dislocations pile up at internal obstacles, sessile dislocations, and grain boundaries. These pile-ups cause back stresses on dislocation sources, making further dislocation emission more difficult. ${ }^{12,27,28}$ Climb of edge dislocations reduces these back stresses and allows additional dislocations to be emitted. Thus dislocation climb is an important softening process at elevated temperatures. Recently this mechanism was introduced in some threedimensional DDD simulations to study coarsening of dislocation loops ${ }^{29-31}$ and in two-dimensional simulations to study the evolution of dislocation cells. ${ }^{32-34}$ In this paper, we investigate the role of dislocation climb in the hightemperature deformation of thin films. We evaluate the effect of climb on the stress-strain curves of thin films and on the dislocation structure that develops during deformation. The paper is organized as follows. In Secs. II and III the mechanism for dislocation climb and the framework of the twodimensional DDD model are reviewed. In Secs. IV and V numerical results are presented and the effect of climb is analyzed. Concluding remarks are presented in Sec. VI.

\section{DISLOCATION CLIMB}

Edge dislocations climb through emission or absorption of point defects. Even though both vacancies and interstitials can cause dislocations to climb, only vacancies are considered in this work because the concentration of interstitials is usually much lower than that of vacancies as a result of their large energy of formation and low energy of migration. ${ }^{35}$ When an edge dislocation is suddenly subjected to a mechanical force, $F_{\mathrm{c}}$, perpendicular to its glide plane, the dislocation starts to climb, emitting or absorbing vacancies in the process. The result is a local departure from the equilibrium vacancy concentration, until eventually the osmotic force associated with the vacancy super- or under-saturation balances the 
mechanical force. At this point, dislocation climb is diffusion controlled and the climb velocity is determined by the flux of vacancies diffusing to or away from the dislocation. If the vacancy concentration reaches its equilibrium value a distance $R$ from the dislocation, the climb velocity can be obtained from the steady-state solution of the diffusion equation with cylindrical

$$
V_{c}=\frac{2 \pi D_{0}}{b \ln (R / b)} \exp \left(-\frac{\Delta E_{s d}}{k_{B} T}\right)\left[\exp \left(\frac{F_{c} \Delta V^{*} / b}{k_{B} T}\right)-1\right] .
$$

Here $b$ is the magnitude of the Burgers vector, $\Delta E_{s d}$ is the vacancy self-diffusion energy, $\Delta V^{*}$ is the vacancy formation volume, which is approximately equal to $b^{3,37,38} k_{B}$ the Boltzmann constant, $T$ the absolute temperature, and $D_{0}$ the pre-exponential diffusion constant. The climb force $F_{\mathrm{c}}$ is taken positive when it favors vacancy emission. Since $R / b$ appears as the argument of a logarithm, its precise value has little effect on the climb velocity and is taken equal to $2 \pi$ in the context of this paper.

When $F_{c} \Delta V^{*} / b \ll k_{b} T$, which is nearly always true except in the vicinity of large stress concentrations, the climb velocity is approximately equal to t9,40 $^{39}$

$$
V_{c} \approx \frac{2 \pi D_{0}}{b \ln (R / b)} \exp \left(-\frac{\Delta E_{s d}}{k_{B} T}\right) \frac{F_{c} \Delta V^{*} / b}{k_{B} T}=\frac{F_{c}}{B_{c}} .
$$

According to Eq. (2), the climb velocity scales linearly with climb force. The proportionality constant represents the climb mobility, which changes rapidly with temperature. Typical values for $B_{c}$ are in the range of $10-10^{3} \mathrm{~Pa} s$ for face-centered cubic (fcc) metals close to their melting point. Equation (2) is valid for a straight dislocation with an axisymmetric diffusion fields.

The equation is derived under the assumption of steadystate vacancy diffusion with a constant far-field vacancy concentration. In actual practice, one would expect the far-field vacancy concentration to evolve with continued dislocation climb, but this effect is not captured by the model. More sophisticated models for dislocation climb that take into account 3D effects such as dislocation jogs or pipe diffusion can be found in Refs. 36, 37, 39, and 41.

\section{DISCRETE DISLOCATION FRAMEWORK}

In discrete dislocation dynamics, a material is generally modeled as an elastic solid containing dislocations. As load is applied to the material, the dislocations are allowed to move and evolve incrementally. At a given instance of time, it is assumed that the material is in equilibrium and that the displacement and stress fields are known. An increment of strain is prescribed and the positions of the dislocations, the displacement field, and the stress field are updated using the following procedure: (1) The Peach-Koehler force is calculated along the length of each dislocation; (2) the dislocation structure is allowed to evolve in response to the PeachKoehler force, including effects such as the nucleation, motion, and annihilation of dislocations; (3) the stress state in the solid is calculated for the updated dislocation arrange- ment. Steps 1 and 3 follow from elasticity; step 2 requires the formulation of constitutive rules for dislocation behavior. In this paper, we follow the rules suggested by Kubin et al. ${ }^{42}$ for dislocation glide, dislocation annihilation and dislocation nucleation.

Determining the stress state at each time step requires the solution of an elastic boundary value problem. In the two-dimensional DDD framework developed by Van der Giessen and Needleman, ${ }^{10}$ the displacement, strain and stress fields are written as the superposition of two fields,

$$
\boldsymbol{u}=\tilde{\boldsymbol{u}}+\hat{\boldsymbol{u}}, \quad \boldsymbol{\varepsilon}=\tilde{\boldsymbol{\varepsilon}}+\hat{\boldsymbol{\varepsilon}}, \quad \boldsymbol{\sigma}=\tilde{\boldsymbol{\sigma}}+\hat{\boldsymbol{\sigma}} .
$$

The $(\sim)$ fields are obtained by summing the fields associated with the individual dislocations in the material under the assumption of an infinite medium,

$$
\tilde{\boldsymbol{u}}=\sum_{I=1}^{N} \boldsymbol{u}^{(I)}, \quad \tilde{\boldsymbol{\varepsilon}}=\sum_{I=1}^{N} \boldsymbol{\varepsilon}^{(I)}, \quad \tilde{\boldsymbol{\sigma}}=\sum_{I=1}^{N} \boldsymbol{\sigma}^{(I)},
$$

where $\boldsymbol{u}^{(I)}, \boldsymbol{\varepsilon}^{(I)}$, and $\boldsymbol{\sigma}^{(I)}$ are the fields due to dislocation $I$, analytical expressions for which can be found in standard texts (see, e.g., Refs. 38 and 39 ). The $(\wedge)$ fields represent the image fields that enforce the correct boundary conditions. They are smooth and are readily calculated using the finite element method or a boundary element analysis. The Peach-Koehler force on a dislocation $I$ is given by

$$
\boldsymbol{F}^{(I)}=\left[\left(\hat{\boldsymbol{\sigma}}+\sum_{J \neq I} \boldsymbol{\sigma}^{(J)}\right) \cdot \boldsymbol{b}\right] \times \boldsymbol{\xi},
$$

where $\boldsymbol{\xi}$ is the local tangent to the dislocation line and $\boldsymbol{b}$ is the Burgers vector. The glide component of this force is $F_{g}^{(I)}=\boldsymbol{F}^{(I)} \cdot(\boldsymbol{\xi} \times \boldsymbol{n})$ and the climb component $F_{c}^{(I)}=\boldsymbol{F}^{(I)} \cdot \boldsymbol{n}$, where $\boldsymbol{n}$ is the unit vector perpendicular to the glide plane of the dislocation given by $\boldsymbol{b} \times \boldsymbol{\xi} /\|\boldsymbol{b} \times \boldsymbol{\xi}\|$.

Simulations typically start with the material in a dislocation-free state. Dislocation sources are randomly distributed on the slip planes with each source characterized by a nucleation strength, $\tau_{\text {nuc }}$. When the glide component of the Peach-Koehler force on a dislocation source exceeds $b \tau_{n u c}$ during a time $t_{n u c}$, two dislocations of opposite sign are nucleated on the glide plane. The distance between the dislocations is taken such that the attraction between the two dislocations is balanced by $\tau_{\text {nuc }}$

$$
L_{n u c}=\frac{\mu}{2 \pi(1-\nu)} \frac{b}{\tau_{n u c}},
$$

where $\mu$ is the shear modulus and $\nu$ is Poisson's ratio of the material.

When dislocations encounter an obstacle, they are pinned until the glide component of the Peach-Koehler force exceeds $b \tau_{\text {obs }}$, where $\tau_{\text {obs }}$ is the strength of the obstacle. When two dislocations of opposite sign come closer to each other than a critical distance $L_{a n n}$, they annihilate each other and are removed from the model.

According to experimental [references in Ref. 43] and computational evidence, ${ }^{44}$ the glide velocity in an fcc 
material without internal obstacles is a linear function of the glide force, i.e., $V_{g}^{(I)}=F_{g}{ }^{(I)} / B$. In this expression, $B$ is called the drag coefficient. The drag coefficient increases linearly with temperature. ${ }^{43}$ In our DDD simulations, we use Eq. (1) to calculate the climb velocity because it is more general than Eq. (2) and can be used in locations with high stresses such as in the vicinity of a dislocation pile up. At each time step, the glide and climb velocities of the dislocations in the simulation are calculated and the positions of the dislocations are updated accordingly. Because the climb velocity is much smaller than the glide velocity, different time steps must be used for climb and glide. In this paper, the time step for climb is taken 100 times larger than the time step for glide.

In an isotropic material, the $x$-component of the displacement field of an edge dislocation with Burgers vector parallel to the $x$-axis is given by ${ }^{38}$

$$
u_{x}=\frac{b}{4 \pi(1-\nu)} \frac{x y}{x^{2}+y^{2}}-\frac{b}{2 \pi} \tan ^{-1}\left(\frac{x}{y}\right) .
$$

For a dipole of edge dislocations in the same glide plane, the displacement field can be obtained from Eq. (7) by superposition. If one of the dislocations in the dipole climbs out of the glide plane, however, simple superposition of the displacement field as given in Eq. (7) does not provide the correct discontinuity in the displacement field. As illustrated in Fig. 1(a), there are indeed discontinuities equal to the Burgers vector on the glide planes, but they do not lie along the path swept out by the dislocations. To correct this shortcoming and to obtain the exact displacement field for a nonplanar edge dislocation dipole, where one of the dislocation in the dipole has climbed from its location in the glide plane $\left(x_{0}, y_{0}\right)$ to a new location $\left(x_{0}, y_{1}\right)$, the following terms need to be added to the displacement in the $x$-direction:

$$
\begin{aligned}
& \frac{b}{2 \pi}\left[\tan ^{-1}\left(\frac{y-y_{1}}{x-x_{0}}\right)-\tan ^{-1}\left(\frac{y-y_{0}}{x-x_{0}}\right)+\tan ^{-1}\left(\frac{x-x_{0}}{y-y_{1}}\right)\right. \\
& \left.-\tan ^{-1}\left(\frac{x-x_{0}}{y-y_{0}}\right)\right] .
\end{aligned}
$$

This extra term accounts for the displacement associated with vacancies emitted or absorbed by the dislocation. The corrected displacement, obtained from the appropriate superposition of displacement fields given by Eq. (7) and the terms in Eq. (8), as shown in Fig. 1(b). It is evident from the figure that the discontinuity in the displacement field now extends exactly along the path of the dislocations.

\section{THIN-FILM MODEL AND SELECTION OF PARAMETERS}

Simulations were performed on freestanding polycrystalline films that were passivated on one or both surfaces and that were subjected to uniaxial tension as illustrated schematically in Fig. 2. In line with Nicola et al., ${ }^{12}$ the film was modeled as a two-dimensional array of rectangular grains of thickness $h$. In doing so, a periodic unit-cell of width $w$ consisting of six randomly oriented grains of uniform size $d$ was considered. Plane-strain conditions were assumed normal to
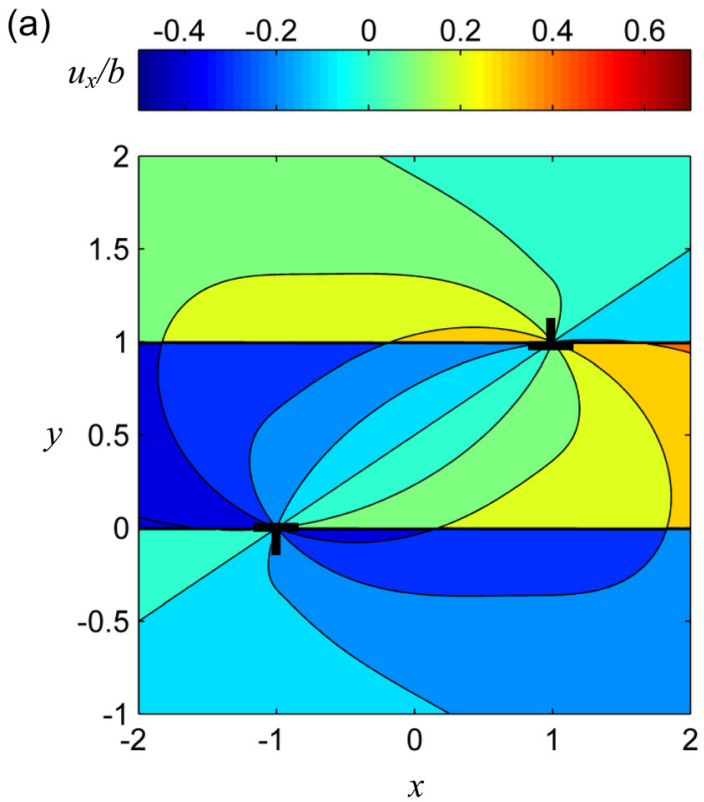

(b)
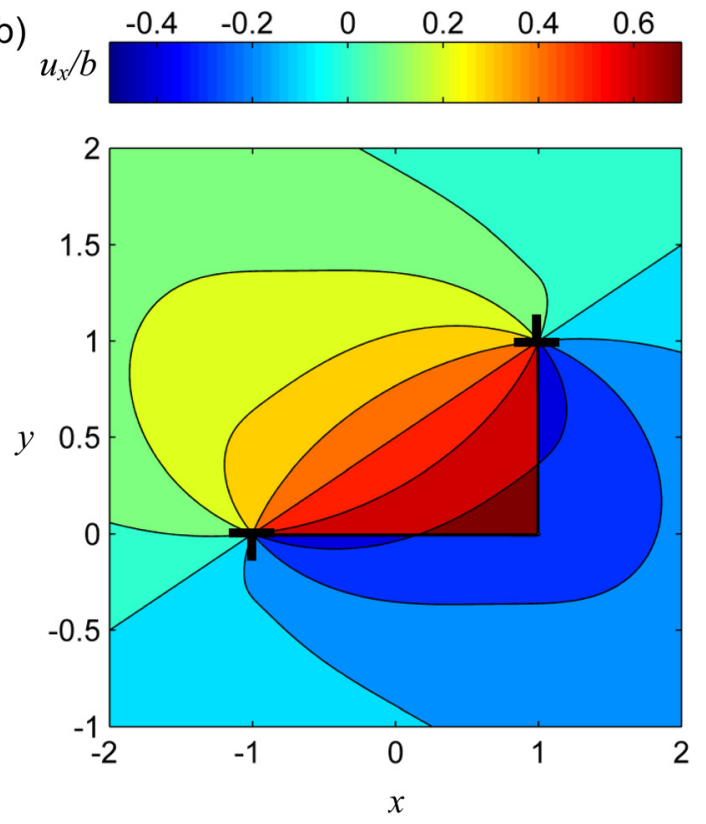

FIG. 1. Displacement field $u_{x}$ (normalized with $b$ ) associated with a dislocation dipole when one dislocation climbs from $(1,0)$ to $(1,1)$ and the other dislocation is positioned at $(-1,0)$. (a) Computed directly from Eq. (7) and (b) extra terms described by Eq. (8) are added.

the $x y$-plane. Grain boundaries and passivation layers were assumed impenetrable to dislocations. Each grain had three sets of slip planes that differed by $60^{\circ} .{ }^{45}$ As mentioned earlier, the grains were initially dislocation free, but FrankRead sources and obstacles were distributed randomly on the slip planes in the grains. Tension was imposed by prescribing a constant displacement rate difference between the left and right edges of the super-cell. The top and bottom surfaces of the super-cell were taken to be traction-free. The average stress in the film, $\sigma$, is calculated as

$$
\sigma=\frac{1}{h} \int_{h} \sigma_{x x}(w, y) \mathrm{d} y,
$$




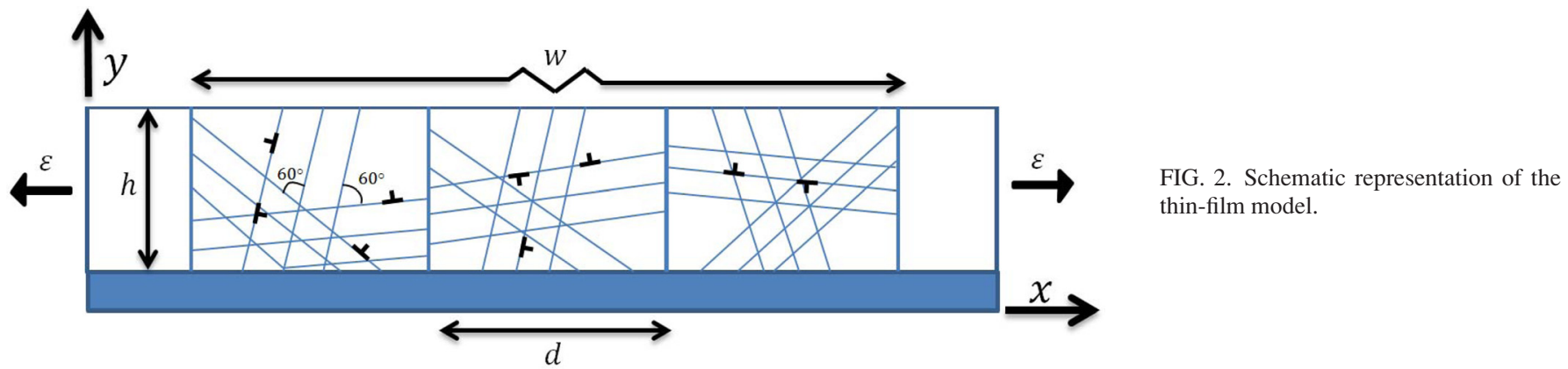

where the integral over the film thickness excludes any passivation layer.

The physical properties used for the film material are representative of aluminum and are given in Table I. The passivation layers were assumed to remain elastic and had the same elastic properties as the film material. The thickness of the passivation layers was $20 \mathrm{~nm}$; the grain size was $1.5 \mu \mathrm{m}$. All simulations were run at a temperature of $800 \mathrm{~K}$. Based on molecular dynamics simulations by Olmsted et al. ${ }^{46}$ the drag coefficient for an edge dislocation in aluminum was estimated at $12 \mu \mathrm{Pa} \mathrm{s}$ at $300 \mathrm{~K}$, yielding a drag coefficient of approximately $32 \mu \mathrm{Pa}$ s at $800 \mathrm{~K}$. The annihilation distance $L_{\text {ann }}$ and the nucleation time $t_{\text {nuc }}$ were chosen as $6 b$ and $10 \mathrm{~ns}$, respectively. The density of dislocation sources was taken as $30 \mu \mathrm{m}^{-2}$ in all simulations. The source strength $\tau_{\text {nuc }}$ was randomly chosen from a Gaussian distribution of strengths with an average value of $100 \mathrm{MPa}$ and a standard deviation of $20 \mathrm{MPa}$. The density of obstacles was $20 \mu \mathrm{m}^{-2}$ and their strength was taken as $150 \mathrm{MPa}$. Because climb allows dislocations to leave their glide planes, dislocations can now occur on all possible glide planes in the material, not just those with dislocation sources. The spacing between glide planes was taken equal to $b$ in all simulations. If the climb distance in a given time step was smaller than the spacing between the glide planes, the dislocation is not allowed to climb in that time step. To limit the computation time, a high strain rate $\dot{\varepsilon}=40001 / \mathrm{s}$ was used for all simulations. To reduce the statistical effects of the initial conditions, several realizations of the model were run for each set of parameters. Realizations differ from each other with respect to the locations of dislocation sources and obstacles.

\section{RESULTS AND DISCUSSION}

Curves of average stress in the film versus imposed strain are depicted in Fig. 3. Each curve is the average of four realizations. Figure 3(a) shows stress-strain curves for

TABLE I. Material properties taken in the simulations.

\begin{tabular}{lccc}
\hline \hline Parameter & Value & Parameter & Value \\
\hline$\mu$ & $26 \mathrm{GPa}^{\mathrm{a}}$ & $\Delta E_{s d}$ & $1.28 \mathrm{eV}^{\mathrm{b}}$ \\
$\nu$ & $0.35^{\mathrm{a}}$ & $D_{0}$ & $0.1185 \mathrm{~cm}^{2} / \mathrm{s}^{\mathrm{b}}$ \\
$b$ & $2.86 \AA^{\mathrm{c}}$ & & \\
\hline \hline
\end{tabular}

${ }^{\mathrm{a}}$ Reference 39 .

${ }^{\mathrm{b}}$ Reference 35 .

${ }^{\mathrm{c}}$ Reference 43. films with one or two passivation layers, while Fig. 3(b) demonstrates the effect of film thickness for films with both sides passivated. In both figures, significantly higher stress levels are observed when dislocations are able to only glide than when they can both glide and climb. The stress-strain curves all exhibit a linear segment in which deformation is elastic. Macroscopic yield occurs at a stress level in the $130-150 \mathrm{MPa}$ range depending on film thickness, and is
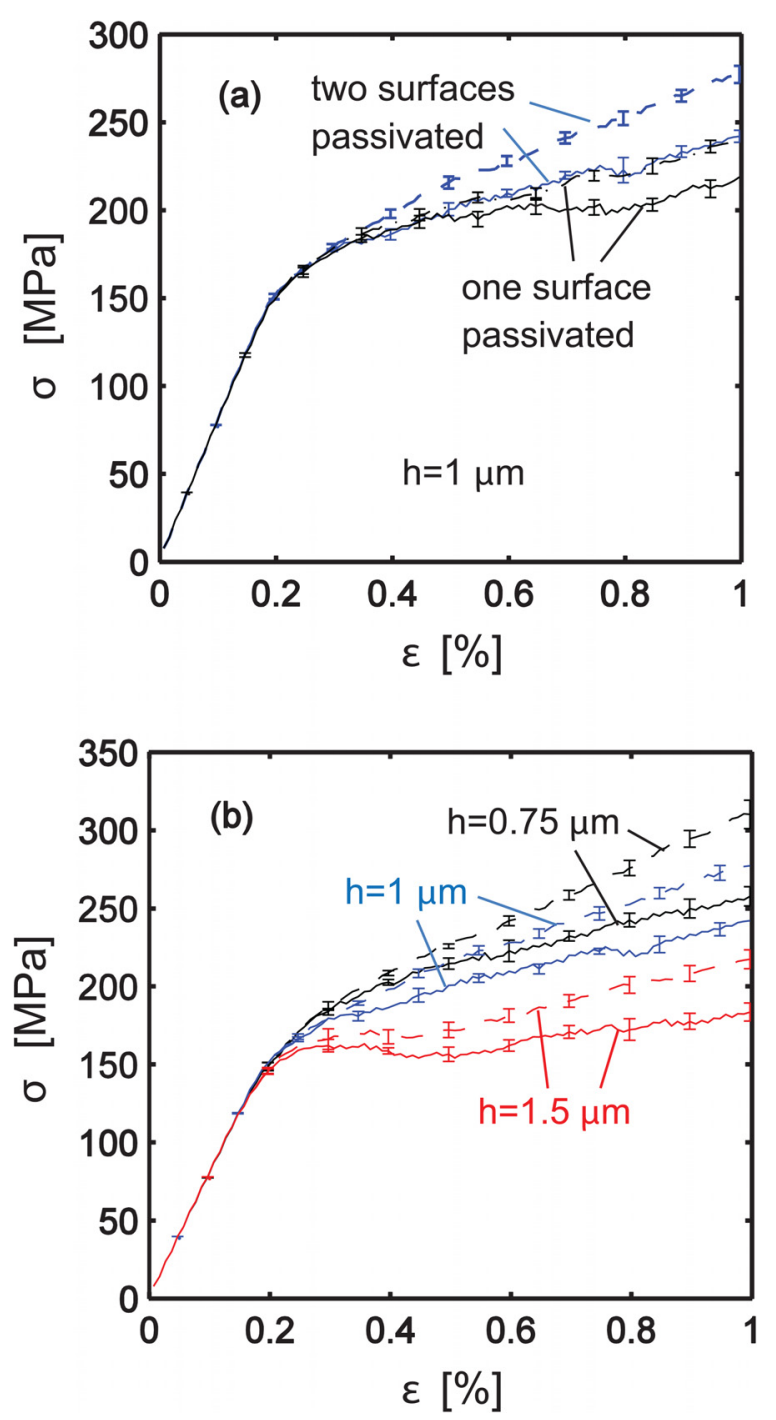

FIG. 3. Stress-strain curves for films. (a) shows the effect of passivation layer(s) for a $1 \mu \mathrm{m}$ film; (b) shows the effect of the film thickness for films with passivation layers on both surfaces. Dashed curves are pertinent to the case of glide only. All curves are obtained as the average of four realizations; error bars represent the standard error for the four realizations. 
followed by a regime in which the film work hardens. The initial parts of the stress-strain curves in Fig. 3(a) - up to a strain of approximately $0.3 \%$ - overlap independent of the surface conditions of the film or the precise deformation mechanism; beyond this strain, the stress-strain curves diverge rapidly. Evidently, for a given film thickness the very first stage of plastic deformation is not strongly dependent on whether one or both surfaces are passivated or whether dislocation climb is allowed; given that the grains are initially dislocation-free, it is more important to nucleate dislocations and this depends primarily on the strength of the available dislocation sources. In the work-hardening regime, by contrast, the flow stress depends quite sensitively on passivation conditions and the availability of a recovery process; Figure 3(a) shows that the flow stress of a thin film increases if both surfaces are passivated and decreases if dislocation climb is allowed. Figure 3(b) illustrates the effect of film thickness: The hardening rate increases with decreasing film thickness. Dislocation climb reduces the hardening rate and this effect becomes more pronounced as the film thickness decreases, presumably because the stress levels, and hence the climb forces, increase with decreasing film thickness.

Figure 4 shows the stress distribution and the dislocation structure at a strain of $1 \%$ for a $1 \mu \mathrm{m}$ film passivated on both surfaces. Figures 4(a) and 4(b) show exactly the same realization except that in Fig. 4(a) dislocation climb is not allowed, whereas in Fig. 4(b) it is. The number of dislocations in the realization with glide only is approximately $10 \%$ higher than in the realization with glide and climb. This trend of a reduction in the number of dislocations if climb is allowed is observed systematically in all simulations. The lower dislocation density in films where climb is allowed

(a)
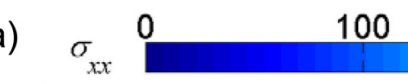

$100 \quad 200$ 300 400 500 [MPa]

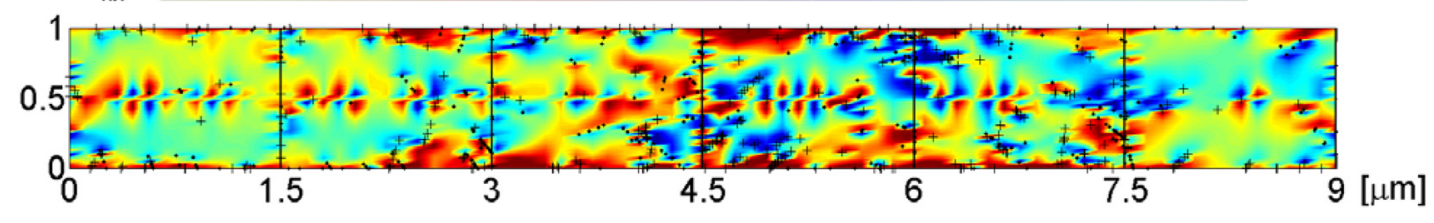

(b) $\sigma_{x x}$

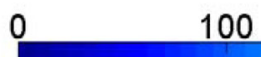

200 300 400 $500[\mathrm{MPa}]$

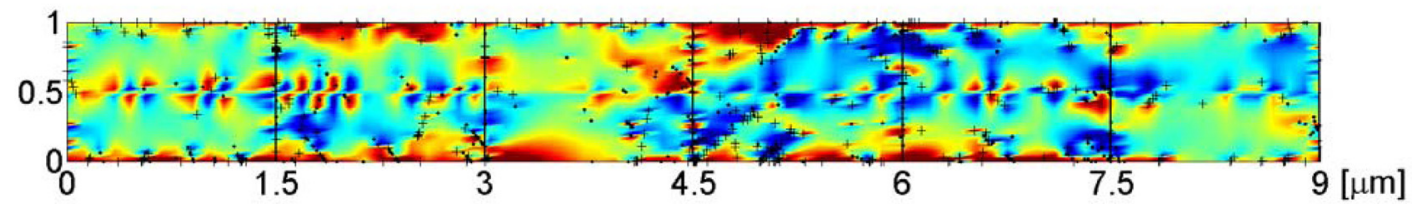

(c)

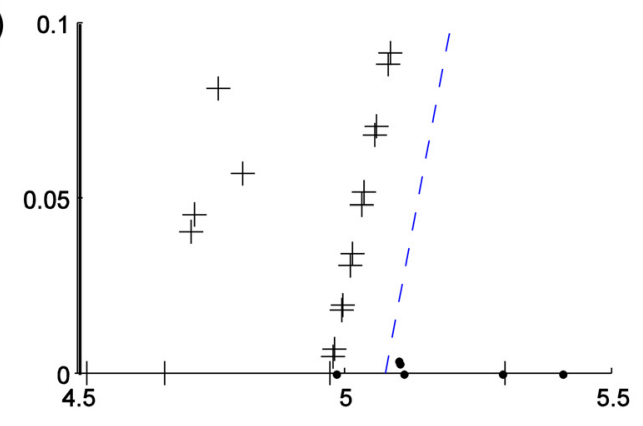

(d)

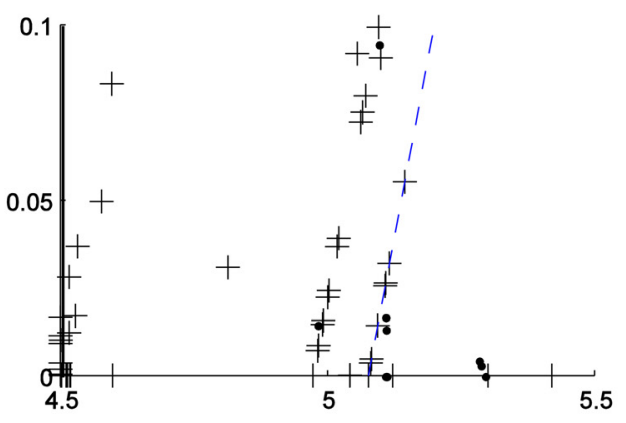

FIG. 4. Distribution of $\sigma_{x x}$ and dislocations at $\epsilon=1 \%$ for a $1 \mu \mathrm{m}$ film when dislocations can (a) glide only and (b) glide and climb. Magnified view of a specific region for (c) glide only and (d) glide with climb. Positive and negative dislocations are marked by the + symbols and solid circles, respectively. 
makes sense: Climb enables dislocations to travel on average a greater distance before they get stuck. By the Orowan equation, the same plastic strain requires a lower dislocation density, as observed. Dislocation climb leads to the formation of new dislocation structures: Figures 4(c) and 4(d) zoom in on a specific region of Figs. 4(a) and 4(b). The slip plane marked by the dashed line in Fig. 4(d) does not contain any dislocation sources, yet it clearly features a dislocation pile-up; the same plane in the realization without climb does not have any dislocations, Fig. 4(c). Thus climb allows dislocations in a pile-up to migrate to a new slip plane and form a new pile up.

It is evident from the stress-strain curves in Fig. 3 that the work hardening rate is reduced as a consequence of dislocation climb. To relate this observation to the dislocation structure that develops in the films, Figs. 5(a) and 5(b) show the average density of pile-ups and the average density of dislocations in pile-ups as a function of film thickness for a film passivated on both surfaces. It is evident from the figures that both quantities decrease when dislocation climb is enabled. In addition, dislocations are dispersed over a larger number of slip planes. Fig. 5(c) shows that the number of slip planes with dislocations (active slip planes) increases more rapidly if dislocations are allowed to climb.

Pile-ups cause back stresses that make it more difficult for dislocations on the same glide plane to move and may even prevent further dislocation emission from Frank-Read sources. ${ }^{12}$ Both effects cause an increase in the work hardening rate. Recovery processes, on the other hand, lead to softening of the material through rearrangement and annihilation of dislocations. In the current model, recovery occurs through climb: Climb disperses dislocations, reduces the number of pile-ups, and decreases the internal stresses. Discrete dislocation modeling in Ref. 12 has shown that when the film thickness decreases or when an additional passivation layer is added, the hardening rate increases with decreasing film thickness or with the addition of a passivation layer because more dislocation pile-ups are formed. Because climb is promoted at higher stress levels and because it reduces the impact of pile-ups, the effects of climb are felt increasingly with decreasing film thickness or in the presence of passivation layers. For the same reasons, one would expect climb to be more prominent when the grain size is smaller or the density of dislocation sources is reduced. ${ }^{12,13}$

In our 2D simulations, dislocation sources and obstacles are regarded as static entities, while in reality they evolve as the dislocation structure changes. Any effects due to the curvature of the dislocations are also not taken into account. Accurate representation of these effects would require fullfledged 3D simulations. To the best of our knowledge, there are currently no measurements of thin-film stress-strain curves in the temperature range where deformation by dislocation climb is significant, although several creep experiments ${ }^{47-52}$ in which the inelastic strain is mainly due to diffusional and/or dislocation (power-law) creep have been reported. We expect the effects of dislocation climb to be more important in films with large grains subjected to high levels of stress, especially when a thin passivation layer suppresses surface diffusion. ${ }^{51}$ The authors are preparing an (a)

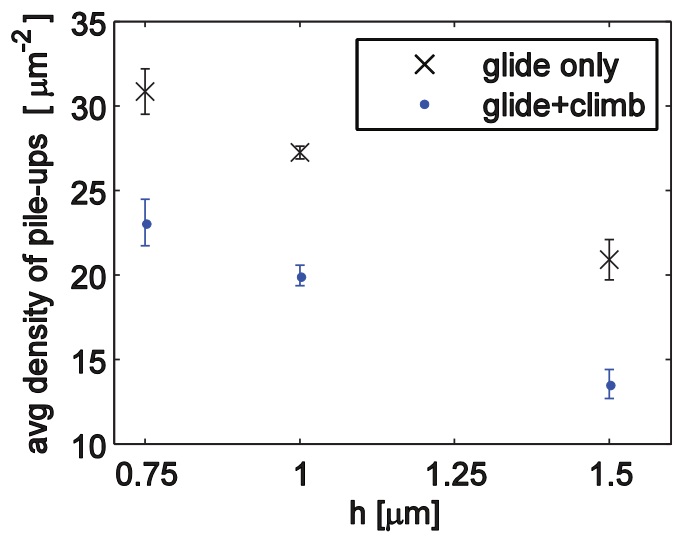

(b)

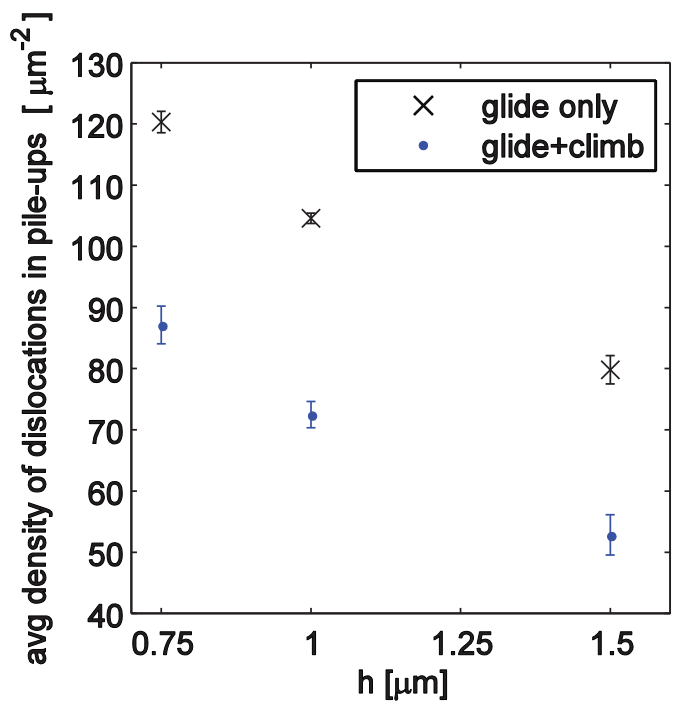

(c)

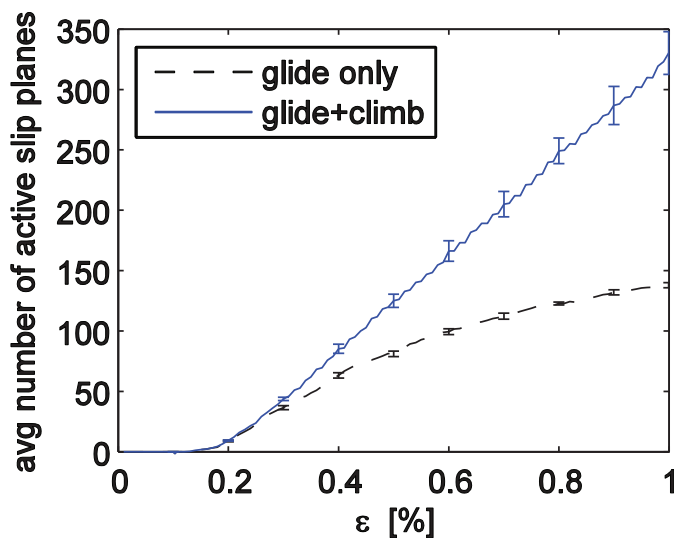

FIG. 5. (a) Average density of dislocation pile-ups and (b) average density of dislocations in pile-ups as a function of film thickness. A pile-up is defined as a collection of same-sign dislocations on a glide plane with a spacing of less than $40 \mathrm{~b}$. (c) Evolution of the number of slip planes containing dislocations during simulations for a $1 \mu \mathrm{m}$ film passivated on both sides. Results are averaged over four realizations; the error bars show the standard errors for the realizations.

experimental setup to measure the stress-strain curves of thin films at elevated temperatures to confirm this conjecture.

\section{CONCLUSIONS}

The present work extends the use of two-dimensional DDD to elevated temperatures, where dislocations can move 
by climb in addition to glide. Using this framework, we have investigated the effect of climb on the response of a polycrystalline aluminum thin film under uniaxial tension. Dislocation climb reduces the flow stress of the film. This effect becomes more pronounced with decreasing film thickness and with the presence of a passivation layer. Because of dislocation climb, dislocations are more dispersed, fewer pile ups form and the overall number of dislocations in pile-ups is smaller.

\section{ACKNOWLEDGMENTS}

The authors are grateful for financial support from the National Science Foundation (Grant No. DMR-0906892) and would like to thank Professor Schöck of Universität Wien for insightful discussions.

${ }^{1}$ N. A. Fleck, G. M. Muller, M. F. Ashby, and J. W. Hutchinson, Acta Metall. Mater. 42, 475 (1994)

${ }^{2}$ J. S. Stölken and A. G. Evans, Acta Mater. 46, 5109 (1998).

${ }^{3}$ N. A. Stelmashenko, M. G. Walls, L. M. Brown, and Y. V. Milman, in Mechanical Properties and Deformation Behavior of Materials Having Ultra-Fine Microstructures, edited by M. Nastasi, D. M. Parkin, and H. Gleiter (NATO ASI Series E 233, 1993), pp. 605-610.

${ }^{4}$ Y. Xiang and J. J. Vlassak, Acta Mater. 54, 5449 (2006).

${ }^{5}$ Y. Xiang, T. Y. Tsui, and J. J. Vlassak, J. Mater. Res. 21(6), 1607 (2006).

${ }^{6}$ Y. Xiang, X. Chen, and J. J. Vlassak, J. Mater. Res. 20(9), 2360 (2005).

${ }^{7}$ J. R. Greer, W. C. Oliver, and W. D. Nix, Acta Mater. 53, 6 (2005).

${ }^{8}$ J. R. Greer and W. D. Nix, Phys. Rev. B 73, 24 (2006).

${ }^{9}$ J. Y. Kim and J. R. Greer, Appl. Phys. Lett. 93, 101916 (2008).

${ }^{10}$ E. Van der Giessen and A. Needleman, Modell. Simul. Mater. Sci. Eng. 3, 689 (1995).

${ }^{11}$ L. Nicola, E. Van der Giessen, and A. Needleman, Thin Solid Films 479, 329 (2005).

${ }^{12}$ L. Nicola, Y. Xiang, J. J. Vlassak, E. Van der Giessen, and A. Needleman, J. Mech. Phys. Solids 54, 2089 (2006).

${ }^{13}$ R. Kumar, L. Nicola, and E. Van der Giessen, Mater. Sci. Eng., A 527, 7 (2009).

${ }^{14}$ R. Kumar, F. Székely, and E. Van der Giessen, Comput. Mater. Sci. 49, 46 (2010).

${ }^{15}$ S. S. Shishvan, L. Nicola, and E. Van der Giessen, J. Appl. Phys. 107, 093529 (2010).

${ }^{16}$ S. S. Shishvan, S. Mohammadi, M. Rahimian, and E. Van der Giessen, Int. J. Solids Struct. 48, 374 (2011).

${ }^{17}$ S. S. Shishvan and E. Van der Giessen, J. Mech. Phys. Solids 58, 678 (2010).

${ }^{18}$ C. Ayas and E. Van der Giessen, Philos. Mag. 88, 3461 (2008).

${ }^{19}$ S. S. Chakravarthy and W. A. Curtin, J. Mech. Phys. Solids 58, 625 (2010).

${ }^{20}$ A. Benzerga, Y. Bréchet, A. Needleman, and E. Van der Giessen, Modell. Simul. Mater. Sci. Eng. 12, 159 (2004).
${ }^{21}$ D. Balint, V. S. Deshpande, A. Needleman, and E. Van der Giessen, Int. J. Plast. 24, 2149 (2008).

${ }^{22}$ A. Needleman, E. Van der Giessen, and V. S. Deshpande, Scr. Mater. 54, 729 (2006).

${ }^{23}$ A. Arsenlis, W. Cai, M. Tang, M. Rhee, T. Oppelstrup, G. Hommes, T. G. Pierce, and V. V. Bulatov, Modell. Simul. Mater. Sci. Eng. 15, 553 (2007).

${ }^{24}$ V. V. Bulatov, L. L. Hsiung, M. Tang, A. Arsenlis, M. C. Bartelt, W. Cai, J. N. Florando, M. Hiratani, M. Rhee, G. Hommes, T. G. Pierce, and T. D. de la Rubia, Nature 440, 1174 (2006).

${ }^{25}$ J. A. El-Awady, S. I. Rao, C. Woodward, D. M. Dimiduk, and M. D. Uchic, Int. J. Plast. 27, 372 (2011).

${ }^{26}$ D. Caillard and J. L. Martin, Thermally Activated Mechanism in Crystal Plasticity (Elsevier Science, 2003).

${ }^{27}$ J. Weertman, J. Appl. Phys. 26, 1213 (1955).

${ }^{28}$ G. Schoeck, "Theory of creep," in Creep and Recovery (American Society for Metals, Cleveland, Ohio, 1957), pp. 199-226.

${ }^{29}$ D. Mordehai, E. Clouet, M. Fivel, and M. Verdier, Philos. Mag. 88, 899 (2008).

${ }^{30}$ D. Mordehai, E. Clouet, M. Fivel, and M. Verdier, IOP Conference Series: Materials Science and Engineering (IOP, 2009), Vol. 3, p. 012001.

${ }^{31}$ B. Bakó, E. Clouet, L. M. Dupuy, and M. Bletry, Philos. Mag. 91, 3173 (2011).

${ }^{32}$ B. Bakó, I. Groma, G. Györgu, and G. T. Zimányi, Comput. Mater. Sci. 38, 22 (2006).

${ }^{33}$ B. Bakó and W. Hoffelner, Phys. Rev. B 76, 214108 (2007).

${ }^{34}$ B. Bakó, I. Groma, G. Györgu, and G. T. Zimányi, Phys. Rev. Lett. 98, 075701 (2007).

${ }^{35}$ Landolt-Beornstein: Numerical Data and Functional Relationship in Science and Technology, edited by O. Madelung (Spinger-Verlag, Berlin, 1990), Vol. 25 of III.

${ }^{36}$ G. Schöck, in Dislocations in Solids, edited by F. R. N. Nabarro (NorthHolland, 1980), Vol. 3, pp. 63-163.

${ }^{37}$ J. Friedel, Dislocations (Pergamon, 1967).

${ }^{38}$ F. R. N. Nabarro, Theory of Crystal Dislocations (Dover, 1987).

${ }^{39}$ J. P. Hirth and J. Lothe, Theory of Dislocations (Wiley, 1982).

${ }^{40}$ H. M. Zbib, M. Rhee, and J. Hirth, Int. J. Mech. Sci. 40, 113 (1997).

${ }^{41}$ R. W. Balluffi and A. V. Granato, in Dislocations in Solids, edited by F. R. N. Nabarro (North-Holland, 1979), Vol. 4, pp. 1-133.

${ }^{42}$ L. P. Kubin, G. Canova, M. Condat, B. Devincre, V. Pontikis, and Y. Bréchet, Solid State Phenom. 23, 455 (1992).

${ }^{43}$ U. F. Kocks, A. S. Argon, and M. F. Ashby, Thermodynamics and Kinetics of Slip (Pergamon, 1975).

${ }^{44}$ A. Yu. Kuksin, V. V. Stegălov, and A. V. Yanilkin, Dokl. Phys. 53, 287 (2008).

${ }^{45}$ J. Rice, Mech. Mater. 6, 317 (1987).

${ }^{46}$ D. L. Olmsted, L. G. Hector, Jr., W. A. Curtin, and R. J. Clifton, Modell. Simul. Mater. Sci. Eng. 13, 371 (2005).

${ }^{47}$ D. Jossel and F. Spaepen, Acta Metall. Mater. 41, 3007 (1993).

${ }^{48}$ D. Jossel and F. Spaepen, Acta Metall. Mater. 41, 3017 (1993).

${ }^{49}$ H. Huang, "Mechanical properties of free-standing polycrystalline metallic thin films and multilayers," Ph.D. dissertation (Harvard University, 1998).

${ }^{50}$ R. M. Keller, S. P. Baker, and E. Arzt, Acta Mater. 47, 415 (1999).

${ }^{51}$ D. Weiss, H. Gao, and E. Arzt, Acta Mater. 49, 2395 (2001).

${ }^{52}$ K. Gall, N. West, K. Spark, M. L. Dunn, and D. S. Finch, Acta Mater. 52, 2133 (2004). 